\title{
Design and Simulation of a Wireless Sensor Network Greenhouse-Monitoring System Based on $3 \mathrm{G}$ Network Communication
}

\author{
http://dx.doi.org/10.3991/ijoe.v12i05.5736 \\ Y. H. Zhou ${ }^{1}$ and J. G. Duan ${ }^{2}$ \\ ${ }^{1}$ Agricultural University of Hebei, Baoding, Hebei, China \\ ${ }^{2}$ Hebei Normal University, Shijiazhuang, Hebei, China
}

\begin{abstract}
A greenhouse provides a stable and suitable environment for the growth of plants. Temperature and humidity are closely related to plant growth. These factors directly affect the water content of plants and the quality of fruits. To solve the problems in the current monitoring system of greenhouse cultivation, such as complicated wiring, large node power consumption, and so on, this study proposes a wireless sensor network greenhouse-monitoring system based on third-generation network communication for the real-time monitoring of the temperature, humidity, light, and $\mathrm{CO}_{2}$ concentration in a greenhouse. GS1011M is regarded as the core in developing wireless terminal nodes. PC software is used to build a real-time observation platform. Sensor data are received in real time through a wireless communication network to complete the monitoring of the target area. A simulation research is also conducted. Results show that the power dissipation of the greenhouse environmental monitoring system is low, its data accuracy is high, and its operation is stable.
\end{abstract}

Index Terms-3G network communication, greenhouse environment, monitoring system, wireless sensor

\section{INTRODUCTION}

Greenhouse facilities are being rapidly developed and expanded. The environmental conditions of a greenhouse directly affect the growth of crops. Therefore, the realtime monitoring of such type of indoor environment is important. Environmental automatic control technology is an effective means to improve the control technology of a greenhouse environment. The temperature, humidity, light intensity, and $\mathrm{CO}_{2}$ concentration in a greenhouse are the data that should be monitored frequently in modern agriculture. The traditional temperature and humidity monitoring system needs to arrange a large number of measurement cables in a greenhouse. Therefore, this study proposes a wireless sensor network (WSN) greenhousemonitoring system based on the third-generation (3G) network communication. The WSN based on wireless communication technology has the advantages of convenient installation, flexible movement, and high precision. This system enables concerned personnel to conveniently monitor various real-time data of each access point (AP) node and terminal node through the network-monitoring platform. The system also allows convenient monitoring of greenhouse equipment to achieve the best temperature, humidity, light, and air states. In the field of agriculture, WSNs have been mainly used to make decisions in irriga-
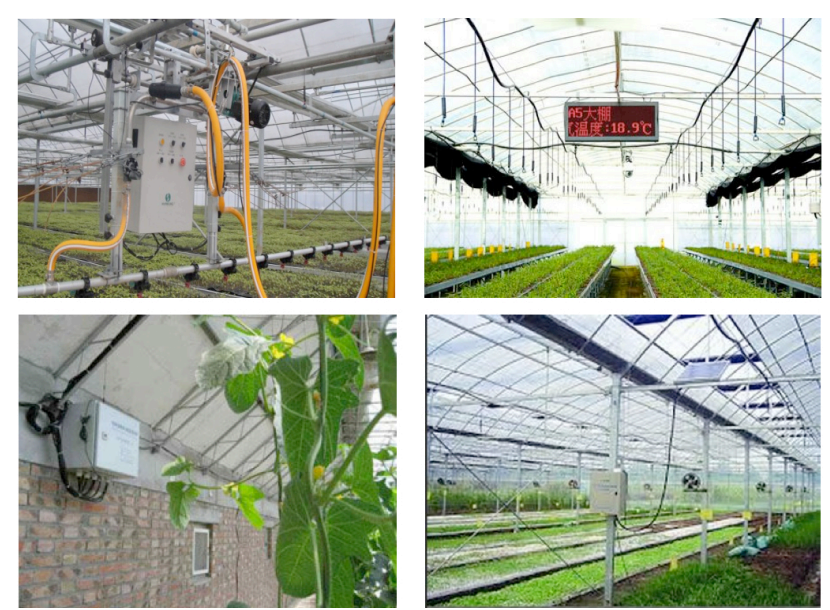

Figure 1. Greenhouse monitoring

tion water resource management and agricultural product storage management, determine crop harvest time, identify crop growth characteristics, and forecast fertilizer demand. Albaladejo.C designed a sensor network topology, including field soil, environment, water, and gateway nodes [1]. O. Green proposed using WSN to monitor temperature change in different positions of a feed bin [2]. Li Li applied WSN to monitor greenhouse environment and established communication with a remote management center through the GPRS mode [3]. Zhang Xiliang realized a WSN based on the ZigBee wireless monitoring system of a greenhouse [4]. Zhou Fu designed a farmland information collection node system based on WSN and combined it with an embedded processor to develop WSN and sink nodes [5]. Vuran M C designed a channel model for electromagnetic wave propagation in soil medium based on WSN [6]. The remainder of this paper is organized as follows. Section 2 describes the related theories and key technologies. Section 3 provides the design and construction process of the proposed WSN greenhousemonitoring system based on $3 \mathrm{G}$ network communication. Section 4 presents a real experiment to evaluate the system. Section 5 concludes the paper.

\section{RELATED THEORIES AND KEY TECHNOLOGIES}

\section{A. Greenhouse Environment Analysis}

Through investigation and analysis, this study determines that the application requirements for a greenhousemonitoring system mainly include the real-time collection 
of environmental factors, such as temperature, humidity, light intensity, and $\mathrm{CO}_{2}$ concentration, among others. Temperature and humidity can be adjusted according to different seeds; greenhouse temperature is generally controlled at $30-20{ }^{\circ} \mathrm{C}$, whereas humidity is generally controlled at $60 \%-90 \% . \mathrm{CO}_{2}$ concentration and light intensity can be adjusted according to seasons and crop growth conditions.

\section{B. Hardware Design of $3 G$ Wireless Communication System Node}

The development of modern communication can be divided into six main periods as follows. First, communication technologies had experienced an initial development from the 1920 s to the 1940 s. For example, the car phone radio system was first installed in St. Louis police cars on June 17, 1946 before it was widely used in North America in the 1980s. Second, the telephone network, which was first established by the Bill System, was installed in public vehicles worldwide, and the related publicly used mobile communication service appeared in the subsequent 20 years. Third, the USA achieved automatic tuning and automatic connection from the 1960s to the 1970s. According to W. Rae Young, Bell Labs invented and realized what could be identified as the first generation (1G) advanced mobile phone system. A cellular mobile communication network was also established. Fourth, on the basis of networks and communications, the UK developed another communication system called Total Access Communication System, which was used in the UK and Ireland in 1983. Fifth, according to Vijay K.G, the Global System for Mobile Communications System was first proposed in Europe in 1982. This system became widely used in most European countries because it could improve voice quality and increase network security. Sixth, according to Patil C.S., Asia and Europe introduced a new communication system called wideband code-division multiple access (WCDMA) at the end of the 20th century, whereas the USA launched CDMA in 2000. These two systems could transmit not only voice but also digital information, which could provide citizens with a convenient and easy access to wireless applications. $3 \mathrm{G}$ mobile communication technology is a cellular mobile communication technology that supports high-speed data transmission. 3G services can transmit voice and data information simultaneously at a general rate of hundreds of bps or higher. The bandwidth of the system is $11 \mathrm{Mbps}$. The system should complete the design and production of WSN nodes, including terminal and AP nodes [7], based on 3G; it provides the sensor parameter values of indoor temperature, humidity, $\mathrm{CO}_{2}$ concentration, and light for the host computer through wireless transmission. $3 \mathrm{G}$ is the third generation of mobile communication standard regulated by the International Telecommunication Union (ITU). 3G provides users with fast Internet access and multimedia service, with which they can talk to one another clearly and easily, as if they are face to face, even at long distances. Compared with second generation (2G)-based digital technology, 3G can provide consumers with faster Internet access speed. This technology was first introduced by the ITU, which concentrated on realizing global roaming. Through this service, people do not have to bring more than one mobile phone when they travel abroad. Accordingly, the entire world can use a particular communication standard. However, several situations, such as conflicts over intellectual property rights and political problems, have resulted in the division of $3 \mathrm{G}$ technologies into three types of technology, namely, W-CDMA, CDMA2000, and time-division synchronous CDMA, which are used in Europe, the USA, and China, respectively. According to Patil C.S., $3 \mathrm{G}$ is backward compatible with $2 \mathrm{G}$ and is accompanied by a series of benefits, such as high-speed Internet access and the realization of video phone and multimedia services, among others. Motorola Solutions states that $3 \mathrm{G}$ expands the bandwidth to the digital subscriber line level, which can ensure speeds ranging from $144 \mathrm{k}$ to $2 \mathrm{M}$. This bandwidth can provide video conferencing as well as fast web browsing and downloading. James $G$ asserted that $3 G$ realizes high transmission speeds and decreased costs that can be acceptable to a wide range of people. $3 \mathrm{G}$ also provides convenience, such as small mobile phone size, long battery life, and good operability.

From the preceding discussion, different ideas can be obtained from three authors. Compared with the conceptual opinions of Patil C.S. and Motorola Solutions, the opinions of James $G$ are more convincing and easier to realize because they provide information on whether $3 \mathrm{G}$ can be used by the general public. The information presented by Motorola Solutions is not sufficiently accurate in terms of transmission speed, considering that $144 \mathrm{k}$ represents speed under special situations, such as moving rapidly. The minimum speed in general situations is $384 \mathrm{k}$. Although $3 \mathrm{G}$ is advanced and convenient, it cannot completely satisfy the requirements of multimedia communication in the field of high resolution.

\section{1) Power module}

The operating voltage of each module of the system is $3.3 \mathrm{~V}$; thus, the power supply uses 2 AA batteries. The power circuit is illustrated in Figure 2.

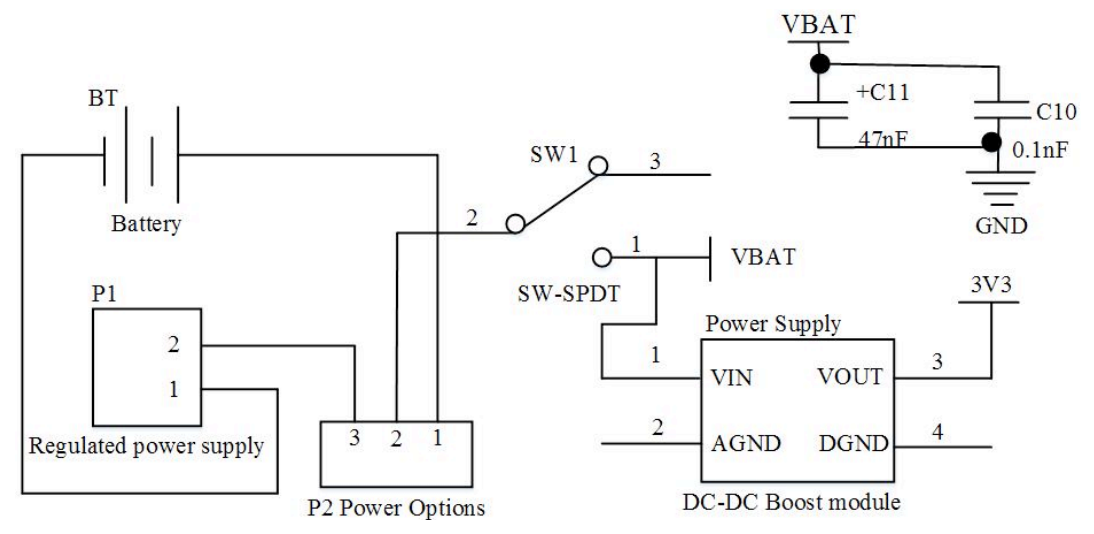

Figure 2. Power supply circuit diagram 


\section{2) $3 G$ module}

With the development of modern society, communication technologies are becoming increasingly essential to human beings. From 1920 to 2013, researchers had made a comprehensive improvement in the fields of voice quality, connection speed, communication security, and so on. In this paper, the main body is divided into three aspects. First, several communication technologies used in history are presented, followed by an overview of technologies used in modern times, which can describe the shift from $1 \mathrm{G}$ to $3 \mathrm{G}$. Second, the advantages and disadvantages of each communication technology are analyzed. Third, a conclusion and a prediction are presented to summarize all the information and suggest possible improvements in the nearest future.

While working on this project, I learn a considerable amount of knowledge that will be valuable for my future study and gain information on how to use resources in the library or on the Internet. My academic knowledge is not yet extensive because I have changed my major from Microelectronics (Undergraduate) to Communication Engineering (Postgraduate); hence, have chosen this project to enrich my knowledge by reading relevant academic journals and books. For example, the unceasingly rising number of websites leads to an increasing demand for fast-speed networks, high resolution when chatting on video phone, or low calling latency. In the future, fourth generation (4G) technology, which is mainly based on orthogonal frequency-division multiplexing (OFDM), will be widely used worldwide. The characteristics of OFDM technology are its highly scalable network structure, good anti-noise performance, and anti-multipath interference capability, which can provide high-quality wireless data technology. A 3G module uses CDMA2000 evolutiondata optimized data transfer unit. CDMA2000 is the $3 \mathrm{G}$ mobile communication used in China; it has the advantages of mature and extensive technologies. In network transmission, CDMA2000 is required to dial and connect to the Internet through the point-to-point (PPP) protocol [8] and then conduct wireless data transmission. PPP protocol dialing is performed by sending the attention command to operate the $3 \mathrm{G}$ module through the controller.

Processor module

The terminal node uses low-power STC89LE52RC subcarrier multiplexing (SCM). The microcontroller input-output port can simulate C2 I interface and sensor module to conduct communication. The power supply voltage is $3.3 \mathrm{~V}$. The AP node does not require a processor.

\section{3) Serial port module}

A 3G module and microcontroller are used through a serial port to conduct communication and through a USB port to perform program configuration, as shown in Figure 3.

\section{4) Sensor module}

A sensor network is a computer network that consists of many automatic devices in spatial distribution. These devices use sensors to cooperatively monitor physical or environmental conditions (e.g., temperature, sound, vibration, pressure, motion, or pollutants) in different locations. The development of WSNs originates from military applications, such as battlefield surveillance. At present, WSNs are used in many fields, such as environmental and eco- logical monitoring, health monitoring, home automation, traffic control, and so on. The traditional sensor is gradually realizing miniaturization, intelligence, information, and network communication. It is experiencing a development process from the traditional sensor (dumb sensor) to the intelligent sensor or embedded web sensor, and is constantly being enriched. With the rapid development of micro-electromechanical system, system on chip, wireless communication, and low-power embedded technology, WSN technology emerges as required by the changing times. This emergence leads to a revolution in information perception with the WSN characteristics of low power consumption, low cost, wide distribution, and selforganization. This technology is becoming a hot spot in various fields. WSN is a cross-disciplinary technology that consists of a large number of inexpensive microsensor nodes deployed in the monitoring area; it is a multihop self-organizing network formed via wireless communication [15]. Microsensor and wireless networking technologies provide an extensive application prospect for WSNs. The potential application areas of WSNs can be summarized as follows: military, aviation, anti-terrorism, explosion, disaster relief, environment, health care, home, industry, business, and other fields. The SHT11 digital temperature and humidity sensor produced by the Swiss Sensirion Company are adopted in this system. The operating voltage is $3.3 \mathrm{~V}$. The serial data line (SDA) and the serial clock line are connected to the microcontroller units (MCUs), namely, P1.2 and P1.3, to conduct communication. The SDA is required to connect to a $10 \mathrm{k} \Omega$ pull resistor to achieve SHT11 control, and thus, read and write temperature and humidity. An ISL29010 digital light intensity sensor is used. The operating voltage is $3.3 \mathrm{~V}$, the working current is $0.25 \mathrm{~mA}$, the standby current is 0.1 $\mu \mathrm{A}$, the measurement precision is \pm 50 lux, and the $\mathrm{P} 2.0$ and $\mathrm{P} 2.1$ are connected to the MCUs. An infrared $\mathrm{CO}_{2}$ sensor is used, the precision of which can reach $10 \times 10^{-6}$. Meanwhile, its power dissipation $<100 \mathrm{~mW}$, and the operating voltage is $3.3 \mathrm{~V}$. The connection electric circuit principle diagram of the sensor and the SCM is shown in Figure 4.

\section{Software Design of $3 G$ Wireless Communication System Node}

In this design scheme, the data collected by multiple terminal nodes are transmitted to the monitoring center through the AP node based on the infrastructure network model and the communication protocol, i.e., transmission control protocol/Internet protocol (IP). In the system, the terminal node regularly sends the collected data to the AP node. The AP node is mainly responsible for transmitting the data frames received from the terminal nodes to the monitoring host. It ultimately provides information, such as temperature, humidity, light, $\mathrm{CO}_{2}$, and other parameters for applications. All nodes in the system work in the same channel, and only one terminal node and one AP node exist for communication; under timer control [9], each terminal node begins to work after being awakened at different times. After the data are collected, they are sent to the AP node, which is configured five times. After sending the data, the timer is full and the sensor is dormant. At other times, both sides are in an unconnected state, and different IP addresses effectively avoid data conflict to reduce the power consumption of the system. Accordingly, the outside world cannot be ignored. The ex- 


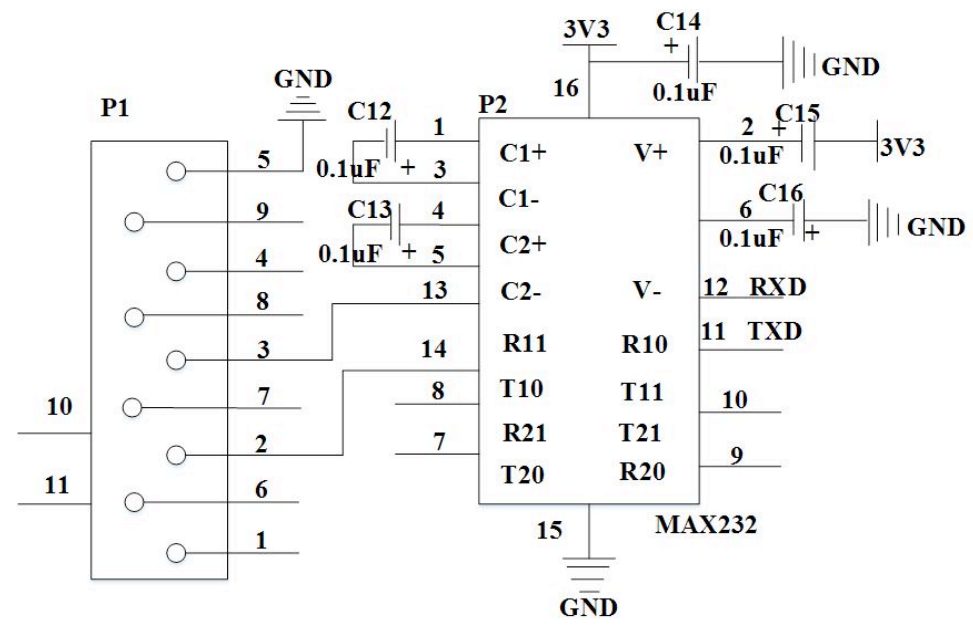

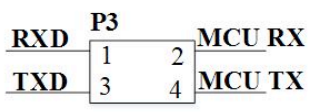

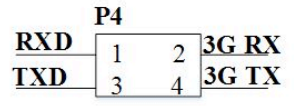

MCU RX $\underset{1}{\text { P5 }}$

Figure 3. Serial interface circuit diagram

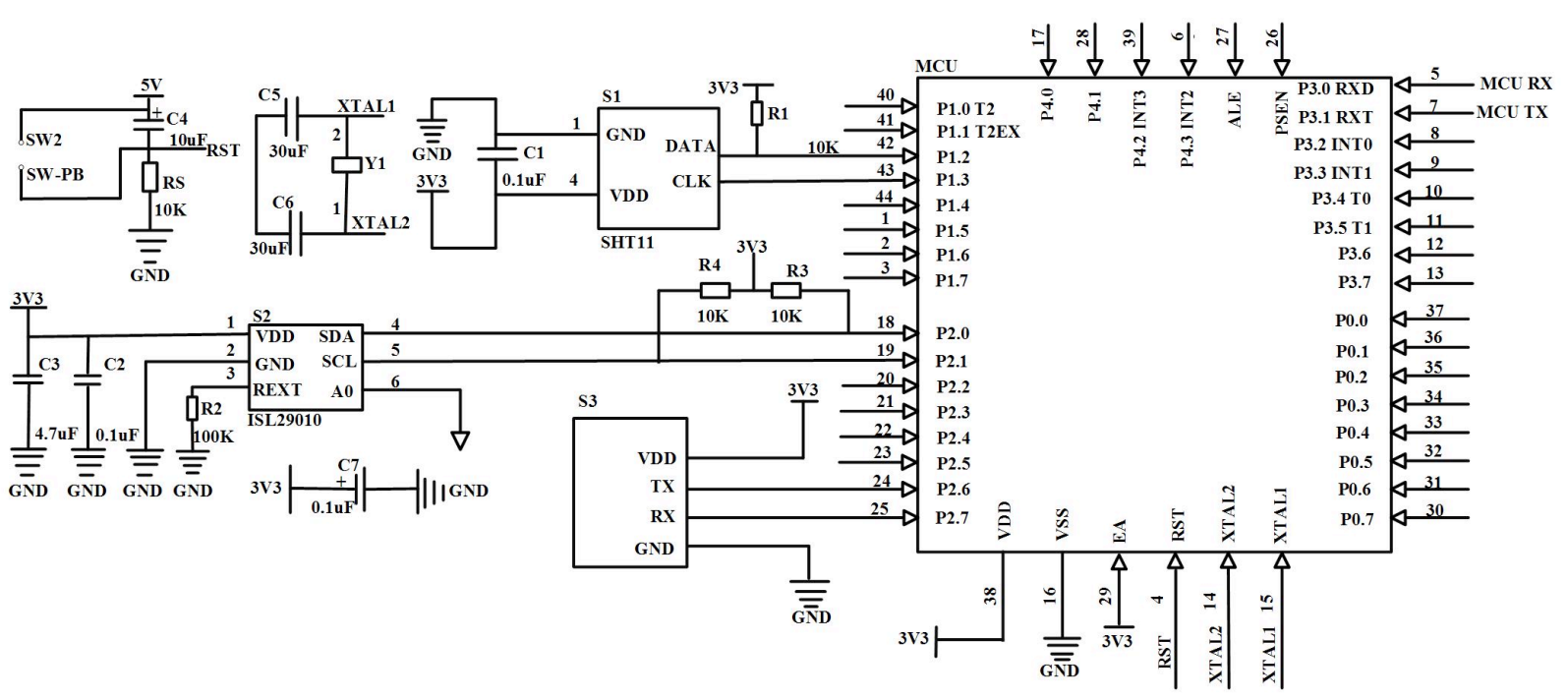

Figure 4. MCU and sensor connection circuit principle

tent of the effect depends on the sensor itself, and thus, the effect can be curbed by improving the sensor. Sometimes, external conditions should be restricted. The main technical indices of error factors are measures of sensor characteristics. Interference inevitably occurs in the actual measurement and control system. External interference in the system is typically called interference, whereas internal interference is called noise. The system will be subjected to various disturbances, such as space electromagnetic wave and strong electric field radiation interference, power frequency interference, $\mathrm{CO}$ interference, and vibration. Noise is usually caused by the internal irregular motion of the generated charged particles. When the electronic switching devices are working, the line current is rapidly changing, which causes noise in the electromagnetic coupling mode with the signal line. In this system, noise is mainly from the conditioning circuit and the electromagnetic switch. Hence, a photoelectric isolator actuator for external interference isolation on the lower computer is used in the design. In the future, $4 \mathrm{G}$, which is mainly based on OFDM, will be widely used worldwide. The characteristics of OFDM technology include a highly scalable network structure, as well as good anti-noise performance and anti-multipath interference capability, which can provide high-quality wireless data technology.

\section{SYSTEM IMPLEMENTATION}

\section{A. System Structure Design}

The overall framework of the greenhouse-monitoring system based on $3 \mathrm{G}$ wireless network is composed of three layers, namely, the collection, data collection, and monitoring center layers.

\section{B. Software Flow of Terminal Nodes}

A PC is the core manager of the entire system; it is mainly composed of serial receiving procedures, a computer management program, and other function modules. The MSCOMM control in Microsoft Visual Studio 2010 is used to design the serial port receiving program, whereas the MFC application frame of the same software is used to design a host computer program [10]. The monitoring center mainly performs sensor setting inquiry, data receiving, data storage, historical data inquiry, and so on. In the measurement and control system, the sensor, signal transmission, and constant current source have different degrees of influence on various factors, which lead to several errors between the instrument display value and the measured true value. Such influences should be eliminated to reduce errors and improve measurement accuracy. Error 
analysis is necessary to enhance the precision of instruments. Factors that affect the measurement accuracy of the system mainly include the following. People always hope that the input and output of a sensor have a definite corresponding relation and exhibit the best linear relationship. However, a linear relationship between output and input requirements is generally inconsistent. The only certainty is that the corresponding input and output cannot be achieved because of the influence of external factors. For different interferences, this study adopts a series of hardware and software anti-interference measures to reduce the effects of disturbance and the instrument itself.

\section{EXPERIMENTAL ANALYSIS}

The system designed in this study is tested on a base. Two nodes are placed in each of four greenhouses, in which the terminal node is 8 , and the routing node is 2 . Temperature and humidity sensors, light intensity sensors, and $\mathrm{CO}_{2}$ sensors are integrated into the terminal node. The terminal node can work for 6 months to 12 months with only 2 ordinary 5 cells. The node is fixed to the ground $1.5 \mathrm{~m}$ in the greenhouse, and the two nodes are spaced apart from the ground by $50 \mathrm{~m}$. The terminal node is sampled once every $30 \mathrm{~min}$ after completing data acquisition and transmission, which automatically enters the sleep state until the subsequent sampling period. Table 1 shows the results of light, temperature, humidity, and $\mathrm{CO}_{2}$ concentration.

TABLE I.

MONITORING RESULT

\begin{tabular}{|c|c|c|c|c|}
\hline $\begin{array}{l}\text { Test } \\
\text { time }\end{array}$ & Temperature $/{ }^{\circ} \mathrm{C}$ & Humidity/\%RH & Light/Lu $x$ & $\mathrm{CO}_{6} / 10^{-}$ \\
\hline $13: 00$ & 25.8 & 72 & 500 & 421 \\
\hline $13: 30$ & 25.9 & 71 & 501 & 432 \\
\hline 14:00 & 26.1 & 71 & 503 & 440 \\
\hline $14: 30$ & 26.1 & 72 & 502 & 456 \\
\hline $15: 00$ & 26.3 & 71 & 499 & 473 \\
\hline $15: 30$ & 26.2 & 71 & 500 & 481 \\
\hline $16: 00$ & 26.1 & 71 & 498 & 501 \\
\hline
\end{tabular}

The results indicate that the power dissipation of the greenhouse environmental monitoring system is low, its data accuracy is high, and its operation is stable. Measurement errors are inevitable, and the device in the measurement circuit has different degrees of errors. The improvement of the accuracy and the repeatability of the test instrument are the objectives that should be achieved.

\section{CONCLUSION}

Greenhouse facilities are rapidly developing and expanding. The environmental conditions in a greenhouse directly affect the growth of crops. Therefore, the realtime monitoring of such indoor environment is important. However, the traditional temperature and humidity monitoring system requires arranging a large number of measurement cables in a greenhouse. To solve this problem, this study proposes a WSN greenhouse-monitoring system based on $3 \mathrm{G}$ network communication. WSNs have become the main approach to realize the remote monitoring of variable information in the agriculture field. This study uses PC software to build a real-time observation platform to complete the monitoring of the target area. A simulation research is also conducted. The greenhouseenvironment monitoring system designed in this study has a good application prospect; it can correctly collect temperature, humidity, light intensity, and $\mathrm{CO}_{2}$ concentration data. Such data can be transmitted through a $3 \mathrm{G}$ network. Nevertheless, the development of WSNs is also facing numerous technical problems. The system designed in this study cannot adjust the greenhouse environment in real time. Adjustment can only be performed after entering the greenhouse. The intelligent control system in the monitoring center should be enhanced to improve the entire system.

\section{REFERENCES}

[1] Albaladejo C, Sánchez P, Iborra A, et al. "Wireless sensor networks for oceanographic monitoring: A systematic review", Sensors, 2010, vol. 10 ,no. 7, pp. 6948-6968. http://dx.doi.org/10.3390/s100706948

[2] O.Green, Nadimi E S, Blanes-Vidal V, et al. "Monitoring and modeling temperature variations inside silage stacks using novel wireless sensor networks", Computers and Electronics in Agriculture, 2009, vol. 69, no. 2, pp. 149-157. http://dx.doi.org/10.1016/j.compag.2009.07.021

[3] $\mathrm{Li} \mathrm{Li,} \mathrm{Li} \mathrm{Haixia,} \mathrm{Liu} \mathrm{Hui,} \mathrm{"Based} \mathrm{on} \mathrm{wireless} \mathrm{sensor} \mathrm{network}$ greenhouse environment monitoring system", Journal of agricultural machinery, 2009, no. S1, pp. 228-231.

[4] Zhang Xiliang, Zhang Weihua, Li Pingping, et al. "Cluster head node of indoor wireless sensor network based on GSM", Journal of Jiangsu University: Natural Science Edition, 2010, vol. 31, no. 2, pp. 196-200.

[5] Park D H, Kang B J, Cho K R, et al. "A study on greenhouse automatic control system based on wireless sensor network", Wireless Personal Communications, 2011, vol. 56, no. 1, pp. 117130. http://dx.doi.org/10.1007/s11277-009-9881-2

[6] Vuran M C, Akyildiz I F. "Spatial correlation-based collaborative medium access control in wireless sensor networks", Networking, IEEE/ACM Transactions on, 2006, vol. 14, no. 2, pp. 316-329.

[7] Malaver A, Motta N, Corke P, et al. "Development and integration of a solar powered unmanned aerial vehicle and a wireless sensor network to monitor greenhouse gases", Sensors, 2015, vol. 15, no. 2, pp. 4072-4096. http://dx.doi.org/10.3390/s150204072

[8] Zhang Q, Yang X, Zhou Y, et al. "A wireless solution for greenhouse monitoring and control system based on ZigBee technology", Journal of Zhejiang University Science A, 2007, vol. 8, no. 10, pp. 1584-1587. http://dx.doi.org/10.1631/jzus.2007.A1584

[9] Kumar A, Hancke G P. "Energy efficient environment monitoring system based on the IEEE 802.15. 4 standard for low cost requirements", Sensors Journal, IEEE, 2014, vol. 14, no. 8, pp. 2557-2566. http://dx.doi.org/10.1109/JSEN.2014.2313348

[10] Chaudhary D D, Nayse S P, Waghmare L M. "Application of wireless sensor networks for greenhouse parameter control in precision agriculture", International Journal of Wireless \& Mobile Networks, 2011, vol. 3, no. 1, pp. 140-149. http://dx.doi.org/10.5121/ijwmn.2011.3113

\section{AUTHORS}

Y. H. Zhou is with the College of Mechanical and Electrical Engineering, Agricultural University of Hebei,

Baoding, Hebei, China (45446900@qq.com).

J. G. Duan (corresponding author) is with the Institute for Nationalities Attached to Hebei Normal University, Shijiazhuang, Hebei, China (45446900@qq.com).

This work was supported by Baoding Science and Technology Research and Development Project (14ZG004).

Submitted, January, 17, 20016 Published as resubmitted by the authors on May, 16, 2016. 